\title{
USE OF ENDOSPORE-FORMING BACTERIA AS AN ACTIVE OXYGEN SCAVENGER IN PLASTIC PACKAGING MATERIALS
}

Tom Anthierens ${ }^{*}$, Peter Ragaert ${ }^{*}$, Sam Verbrugghe ${ }^{* *}$, Assia Ouchchen ${ }^{* *}$, Johan Mertens ${ }^{* * *}$, Lynda Beladjal $^{* * * *}$, Dirk De Cuyper ${ }^{* * * *}$, William Dierickx ${ }^{* * * *}$, Filip Du Prez ${ }^{* *}$ and Frank Devlieghere $^{*}$

* Ghent University, Faculty of Bioscience Engineering, Department of Food Safety and Food Quality, Laboratory of Food Microbiology and Food Preservation, Coupure Links 653, 9000 Ghent, Belgium.

** Ghent University, Faculty of Sciences, Department of Organic Chemistry, Polymer Chemistry Research Group, Krijgslaan 281 (S4bis), 9000 Ghent, Belgium.

*** Ghent University, Faculty of Sciences, Department of Biology, Terrestrial Ecology Unit, Ledeganckstraat 35, 9000 Ghent, Belgium

***** Resilux nv, Damstraat 4, 9230 Wetteren, Belgium

\begin{abstract}
In this study the use of heat resistant endospore-forming aerobic microorganisms of the genus Bacillus amyloliquefaciens as an active oxygen scavenger in multilayer PET bottles was evaluated. Therefore a modelsystem was developed in which Bacillus amyloliquefaciens spores were incorporated in a PET copolymer (PETG) at $220^{\circ} \mathrm{C}$. The effectiveness of the OS was evaluated directly by measuring the oxygen absorption rate and indirectly by determining the viability of the incorporated spores.
\end{abstract}

\section{Introduction}

The use of plastic packaging materials, composed of for example polyethylene terephthalate (PET), as a container for sensitive food products is often restricted due to the weaker gas barrier properties of these polymers compared to glass. In many cases, the presence of oxygen is unwanted because it is responsible for oxidative detoriation reactions, alteration of color and flavor and spoilage by aerobic micro-organisms of the food content $[1 ; 2]$. The incorporation of active oxygen scavengers (OS) in the polymeric matrix can improve the gas barrier properties of the package and remove residual oxygen. An alternative approach to available chemical OS is the incorporation of aerobic microorganisms, capable of consuming oxygen, in the polymeric matrix [3]. A natural and biological oxygen absorption system based on micro-organisms could have advantages to consumer perception, recyclability, safety, material compatibility, production costs, ... A significant restriction of the use of biological systems is the heat stability of incorporated micro-organisms during the high temperature production process of plastic containers and in this specific case of PET bottles.

In this work heat resistant endospore-forming aerobic micro-organisms (genus Bacillus amyloliquefaciens) were incorporated at high processing temperatures in a polymeric matrix as an active oxygen scavenger (OS) $[4 ; 5]$. 


\section{Material and methods}

\subsection{Spore preparation}

In this study spores of Bacillus amyloliquefaciens LMG ID9698 were used. Dry spore powder was produced by inoculating $1 \mathrm{ml}$ of nutrient broth containing a full grown culture $\left(24 \mathrm{~h}\right.$ incubated at $\left.30^{\circ} \mathrm{C}\right)$ onto petri dishes containing a nutrient rich growth medium $(20 \mathrm{~g} / \mathrm{l}$ peptone, $20 \mathrm{~g} / 1$ sucrose, $10 \mathrm{~g} / 1$ yeast extract and $20 \mathrm{~g} / 1$ agar). Inoculated plates were incubated for 5 days at $37^{\circ} \mathrm{C}$ after which spores were harvested by scraping the surface with sterile spatulas. Scrapings were collected in a sterile petri dish and dried for 2 weeks at $37^{\circ} \mathrm{C}$ after which they were grinded.

\subsection{Spore incorporation}

Spores were incorporated in Poly(ethylene terephthalate, 1,4-cyclohexane dimethanol) (PETG), an amorphous copolymer of terephtalic acid with ethylene glycol and cyclohexane dimethanol. PETG resins were pressed into round samples using a mould and table press. Spores incorporation was done by placing the desired amount of spores between two PETG plates and pressing them at $210^{\circ} \mathrm{C}$ under a maximum pressure of 30 bar for 3 minutes. Spore concentrations are expressed as log spores/g PETG. For example $7 \mathrm{log}$ spores/g PETG means that $1 \mathrm{~g}$ PETG material contains $10^{7}$ spores.

\subsection{Oxygen permeation measurements}

Monolayer plates, produced with a polymer press, were placed between the two chambers of a measurement device (Figure 1). The difference in $\mathrm{PO}_{2}$ was maximized by flushing the upper chamber with 2 bar $100 \% \mathrm{O}_{2}$ and the lower chamber with $100 \% \mathrm{~N} 2$. Permeated $\mathrm{O}_{2}$ was accumulated and measured in the lower chamber with an Oxysense 210T. Addition of water was necessary to promote spore germination.

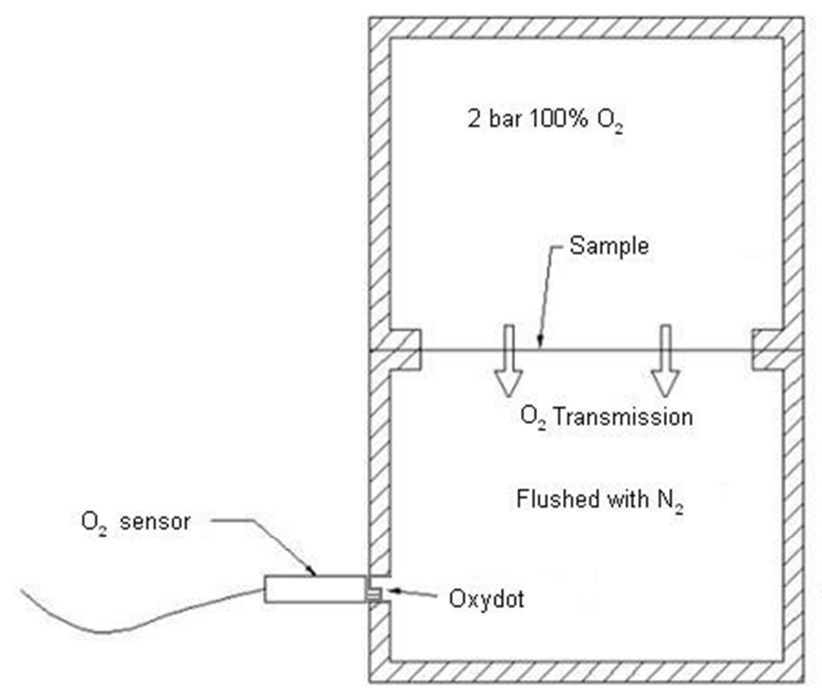

Figure 1. Schematic representation of a high pressure permeation measuring device. Moisture was added on top of the sample to allow spore germination. 


\subsection{Oxygen absorption measurements}

The oxygen absorption rate (OAR $\mathrm{ml} \mathrm{O}_{2} / \mathrm{g} /$ day) was determined by placing monolayer PETG plates in a closed recipient. Water was added in the recipient to allow spore germination. The oxygen concentration in the headspace was measured over time with an Oxysense 210T.

\section{Results and discussion}

Oxygen permeation measurements through samples containing 7.2 log spores/g PETG, 7.9 $\log$ spores/g PETG and $9.4 \mathrm{log}$ spores/g PETG are given in Fout! Verwijzingsbron niet gevonden.. A clear dosis response can be noted between the spore concentration and the oxygen permeation through the sample plates. For samples without spores a linear increase of oxygen over time was found showing no active scavenging. For samples containing 7.2 $\log$ spores/g PETG a minimal effect on oxygen permeation was found compared to a samples containing no spores. These samples also showed a linear increase of oxygen, but at a slightly slower rate. A raise in concentration to $7.9 \mathrm{log}$ spores/g caused an increase of oxygen to $0.36 \%$ after three days. Further measurements showed decrease of the oxygen concentration in the lower measuring chamber meaning that spores were not only able to completely scavenge all permeated oxygen, but also remove already accumulated oxygen. The lower scavenging effect measured in the first three days can be explained by the time needed for moisture uptake, spore activation and germination. Before spores can germinate the minimal conditions needed for growth must be reached. In practice this means that moisture uptake is necessary to reach the minimal water activity $\left(\mathrm{a}_{\mathrm{w}}\right)$ needed.

A further increase of the spore concentration to $9.4 \mathrm{log}$ spores/g PETG showed a similar trend as samples containing $7.9 \mathrm{log}$ spores/g PETG, but with a smaller increase of oxygen during the first days of measurement, and a fast removal of accumulated oxygen. It is noted that after 7 days measurement no significant pressure loss of oxygen was found in the upper chamber, so that it can be stated that measuring oxygen accumulation will have no significant effect on the difference in partial pressure over the sample. 


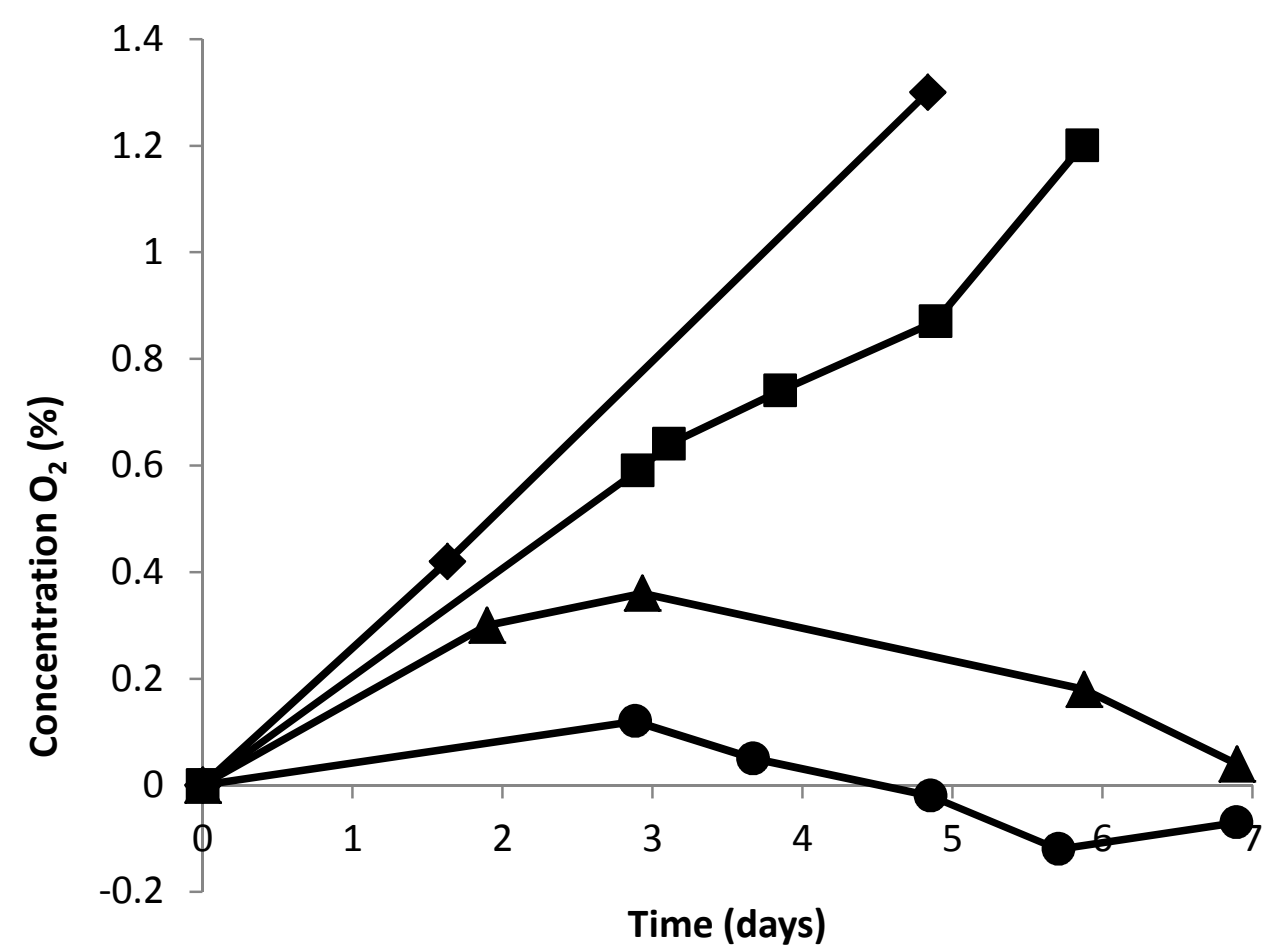

Figure 2. Oxygen permeation through a monolayer plate with low, medium and high spore concentration compared with normal PETG. Permeation was measured through plates without spores $(\diamond)$, with 7.2 log spores/g PETG (घ), with 7.9 log cfu/g PETG ( $\triangle$ ) and with $9.4 \log$.

Next to oxygen permeation, scavenging activity of incorporated spores was tested by measuring the oxygen absorption rate (OAR) of sample plates by placing them in a closed recipient. The OAR of samples containing $9.4 \mathrm{log}$ spores/g PETG were compared with PETG plates containing no spores and with a chemical oxygen scavenger (Amosorb) (Fout! Verwijzingsbron niet gevonden.).

In all measurements with samples containing spores and Amosorb a clear reduction in oxygen could be measured over a period of 7 days. From day 2 to day 7 a linear decrease with $0.04 \pm 0.02 \mathrm{ml} \mathrm{O}_{2}$ /day and $0.03 \pm 0.01 \mathrm{ml} \mathrm{O}_{2} /$ day in oxygen was found for samples containing spores and Amosorb respectively, pointing out that there was no significant difference in OAR between the two. 


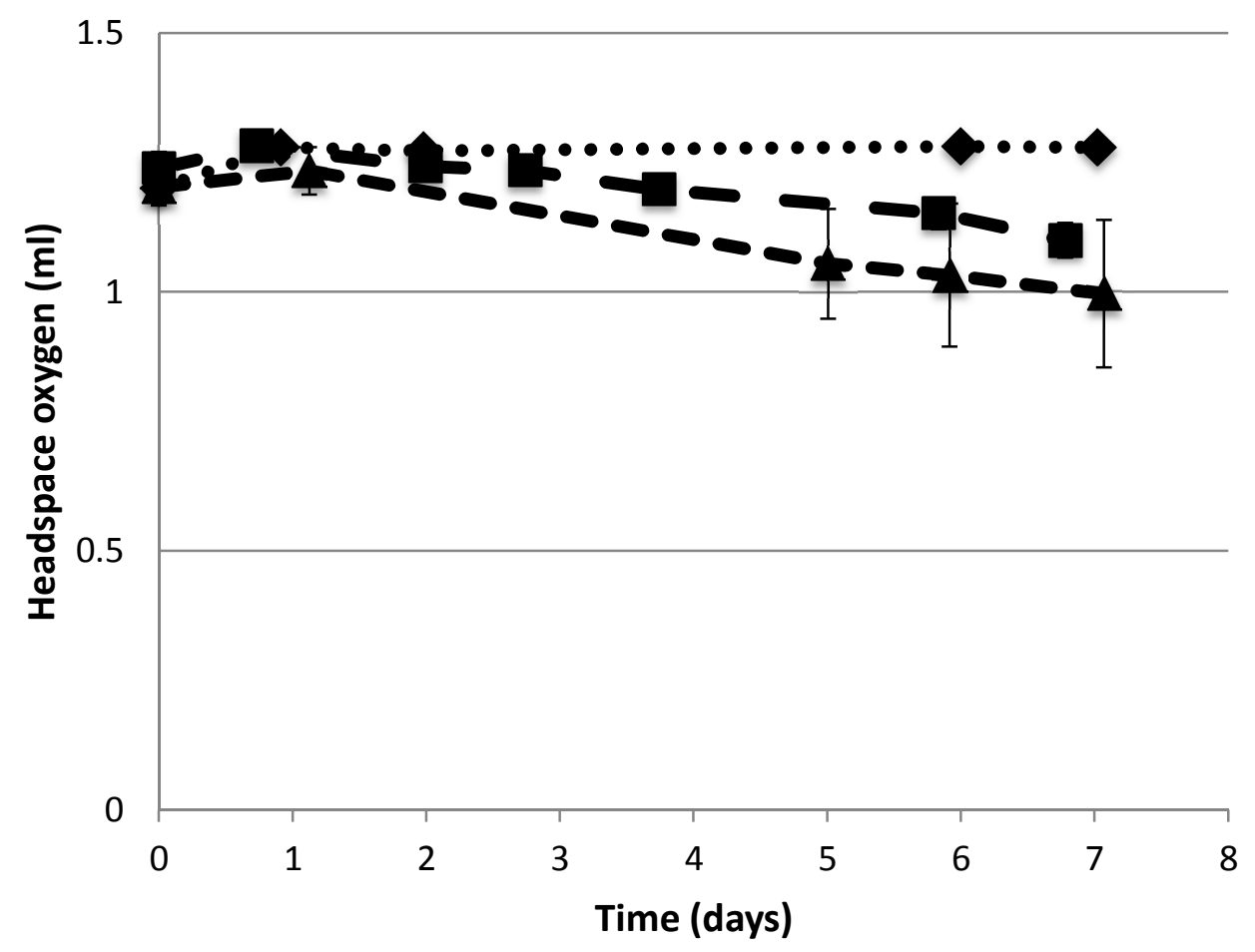

Figure 3. Oxygen absorption by a monolayer plate containing $9.4 \log$ spores/g PETG ( $\Delta$ ) compared with PETG without spores $(\diamond)$ and a commercial OS (Amosorb)(匹).

\section{Conclusions}

This study showed that Bacillus amyloliquefaciens spores can act as an active oxygen scavenger when incorporated in a PETG matrix. Addition of minimum $7.9 \mathrm{log} \mathrm{spores} / \mathrm{g}$ PETG makes the samples net impermeable to oxygen over a period of 7 days with a difference in oxygen partial pressure over the sample of 2 bar. When samples were placed in a closed recipient, an oxygen absorption rate comparable to Amosorb was found.

\section{References}

[1] Ozdemir, M., \& Floros, J. D. (2004). Active food packaging technologies. Critical Reviews in Food Science and Nutrition, 44 (3), 185-193.

[2] Vermeiren, L., Devlieghere, F., van Beest, M., de Kruijf, N., \& Debevere, J. (1999). Developments in the active packaging of foods. Trends in Food Science \& Technology, 10 (3), 77-86.

[3] Altieri, C., Sinigaglia, M., Corbo, M. R., Buonocore, G. G., Falcone, P., \& Del Nobile, M. A. (2004). Use of entrapped microorganisms as biological oxygen scavengers in food packaging applications. Lebensmittel-Wissenschaft und -Technologie, 37 (1), 9-15.

[4] Dierickx, W., De Cuyper, D., Beladjal, L., Mertens, J., Mertens, D., \& Dierickx, Y., Resilux (2006) Process for manufacturing hydrophobic polymers, WO patent 2006/024115.

[5] Mertens, J., Beladjal, L., Devlieghere, F., Verbrugghe, S., Sas, B., Du Prez, F., Anthierens, T., \& Ouchchen, A., Ghent University (2010) Incorporation of thermo-resistant and/or pressure-resistant organisms in materials, WO patent 2010/034776 A1. 